\title{
Investigation of the Unsteady Flow Development over a Pitching Airfoil by Means of TR-PIV
}

\author{
K. Mulleners, A. Henning, H. Mai and M. Raffel \\ DLR, German Aerospace Center, 37073 Göttingen, Germany \\ and \\ A. Le Pape and M. Costes \\ ONERA, 92190 Meudon, France
}

\begin{abstract}
The flow over an OA209 airfoil subjected to a sinusoidal pitching motion under dynamic stall conditions is investigated experimentally by means of time resolved particle image velocimetry (TR-PIV) and surface pressure measurements. Dynamic stall is distinguished by the formation and convection of large scale coherent structures and a delay in massive flow separation. A vortex detection scheme based on an identification function derived directly from the velocity fields is adopted to identify vortex cores. The combination of global time resolved imaging and an automated vortex identification algorithm allows for the investigation of the spatial and temporal evolution of vortical structures within a single oscillation. Furthermore, the mechanisms associated with the dynamic stall delay are considered.
\end{abstract}

\section{Introduction}

$\mathrm{D}$ YNAMIC stall refers to the stall process of an airfoil subjected to an unsteady change in angle of attack, such as a constant pitching motion or a sinusoidal oscillation. The dynamic stall phenomenon has been the subject of numerous investigations over decades ${ }^{1-4}$ and it remains an object of scrutiny for its potential in improving the manoeuvrability and performance of rotary-wing aircraft.

The dynamic stall process comprises a series of complicated aerodynamic phenomena and it is accompanied by a delay in the onset of massive flow separation to higher angles of attack compared to quasi-steady stall onset. The promiment features within the dynamic stall life cycle associated with an oscillating airfoil are consecutively boundary layer separation, the formation and convection of large scale coherent structures, massive flow separation, and finally flow reattachment. Analoguously, Shih et al. ${ }^{5}$ classify the unsteady flow development over an airfoil pitching-up at constant rate into four successive stages, i.e. a vortex formation stage, a vortex convection stage, stall onset and a stalled stage.

Separation of the boundary layer leads to the formation of a local free shear layer, which is then subjected to a Kelvin-Helmholtz type of instability resulting in the formation of small-scale vortical structures. These small structures quickly roll up into large-scale coherent structures, referred to as dynamic stall vortices. Subsequent strong inviscid/visous interactions cause the primary stall vortices to break away from the surface initiating stall. Stall onset is generally accompanied by a loss of lift and an increase of the negative pitching moment. A detailed description of the mechanism causing the stall vortices to be pinched off from the boundary layer can be found in Shih et al. ${ }^{6}$ and Doliganski et al. ${ }^{7}$. The process of vortex formation and convection results in a delay of stall to angles of attack larger than the static stall angle. During this period of time the lift overshoot, which is another characteristic feature of dynamic stall, is produced. The stalled stage is characterized by large scale vortex shedding and corresponding large fluctuations of the lift and the pitching moment.

In order to sustain and to improve dynamic stall modelling and ultimatelly guide dynamic stall control, profound characterisation of the basic features is necessary. Therefore, an investigation of the flow development and vortex dynamics within a dynamic stall life cycle is desirable. From an experimental point of view this requires global time resolved measurement techniques. This paper presents an experimental investigation of the unsteady flow field over an oscillating airfoil by means of time resolved particle image velocimetry 
(TR-PIV) and surface pressure measurement. Emphasis is placed on the spatiotemporal evolution of the vortical structures that emerge with flow separation.

The investigation of vortices and vortex dynamics from experimental data remains a challenging task due to the lack of an accepted definition of a vortex and the difficulty to quantify them. In the literature several specific definitions have been proposed in this context ${ }^{8,9,9,10}$. Due to the difficulty of defining vortices unambiguously, different vortex detection criteria are available, each of them more or less adequat depending on the specific problem. Comprehensive overviews of the diversity of vortex detection schemes and their applications are provided by Jeong and Hussain ${ }^{11}$ and Cucitore ${ }^{12}$. The objective in this paper is not to debate on the adequateness of different vortex detection procedures, but to identify the algorithm that is most suitable for the present application and to focus on the vortex dynamics.

The paper is organised as follows. In the first section the experimental set-up is described. The most commonly used vortex identification schemes are considered in section III, along with the vortex centre identification function adopted for this study. Subsequently, the results of the identification process will be examined in section IV, with regard to the vortex formation, spatiotemporal evolution, dynamic stall onset and delay.

\section{Experimental Methods and Set-Up}

Measurements were performed in the closed-circuit, low-speed wind tunnel at DLR Göttingen. The wind tunnel had an open test section with a rectangular nozzle $(0.7 \mathrm{~m} \times 1.0 \mathrm{~m})$. Free stream velocities up to 55 $\mathrm{m} / \mathrm{s}$ can be reached. The airfoil model was an OA209 airfoil profile with a chord length of $c=0.3 \mathrm{~m}$ and a span of $1 \mathrm{~m}$. The carbon fibre model was equipped with 45 miniature unsteady pressure transducers chordwisely distributed on the suction and pressure side at the model mid-span. A hydraulic pitch-oscillation set-up was arranged symmetrically outside of the wind tunnel test section and the model was oscillated about its quarter chord axis by means of two hydraulic actuators operating in phase opposition. The model motion was sinusoidal according to

$$
\alpha(t)=\alpha_{0}+\alpha_{1} \sin \left(2 \pi f_{\text {osc }} t\right)
$$

where $\alpha_{0}$ is the mean incidence, $\alpha_{1}$ the oscillation amplitude and $f_{\text {osc }}$ the oscillation frequency. For the present study these three parameters were varied with $\alpha_{0} \in\left\{18^{\circ}, 20^{\circ}, 22^{\circ}\right\}, \alpha_{1} \in\left\{6^{\circ}, 8^{\circ}\right\}$, and $f_{\text {osc }} \in\{2.6 \mathrm{~Hz}$, $4.0 \mathrm{~Hz}, 5.3 \mathrm{~Hz}\}$.

The surface pressures were scanned at approximately $6 \mathrm{kHz}$ for about $15 \mathrm{~s}$. Simultaneously three component time resolved particle image velocimetry (TR-PIV) measurements were conducted in the cross sectional plane at model mid-span. The TR-PIV system consisted of a frequency doubled Nd:YAG laser, which supports Q-switch frequencies up to $30 \mathrm{kHz}$ and a pair of CMOS cameras with a $1024 \mathrm{px} \times 1024 \mathrm{px}$ sensor, operating at $3000 \mathrm{fps}$ at full resolution. Series of $3072 \mathrm{PIV}$ image frame pairs were acquired at $1.5 \mathrm{kHz}$ (3000 fps), corresponding to a measurement time of approximately $2 \mathrm{~s}$. The cameras were mounted in a stereoscopic set-up alongside the wind tunnel's diffuser as schematised in figure 1. After mapping the views of both cameras, the dimensions of the PIV measurement window were $284 \mathrm{~mm}$ by $214 \mathrm{~mm}$ and the spatial resolution was $5.0 \mathrm{px} / \mathrm{mm}$. The PIV images were processed using an interrogation window size of $24 \mathrm{px} \times$ $24 \mathrm{px}$ and a $50 \%$ overlap yielding a grid spacing of $12 \mathrm{px}$ or $2.4 \mathrm{~mm}$ which is less then $1 \%$ of the chord length. The resulting images were rotated into the airfoil reference system with the x-axis along the chord, the y-axis along the span and the z-axis upward perpendicular to the chord, where the origin coincides with the leading edge of the airfoil at model mid-span. The free stream velocity was $U_{\infty}=50 \mathrm{~m} / \mathrm{s}$ corresponding to a Reynolds number $R e=9.2 \times 10^{5}$ based on the airfoil's chord length and a Mach number $M a=0.14$. The reduced frequency

$$
k=\frac{\pi f_{\mathrm{osc}} c}{U_{\infty}}
$$

was $k \in\{0.05,0.075,0.10\}$ for the data relevant for this paper.

The described test rig with the OA209 airfoil model was used in the past for unsteady measurements in the transonic wind tunnel at DLR Göttingen ${ }^{13,14}$. The latter has a closed test section and disposes of adaptive upper and lower wind tunnels walls. Quick examination of the results obtained in the different wind tunnels for like boundary conditions reveal a large influence of the open jet flow. The available wind tunnel corrections for open jet flows ${ }^{15}$ are applicable only to fully attached flows. Unfortunately, no proper corrections are available for unsteady flows. 


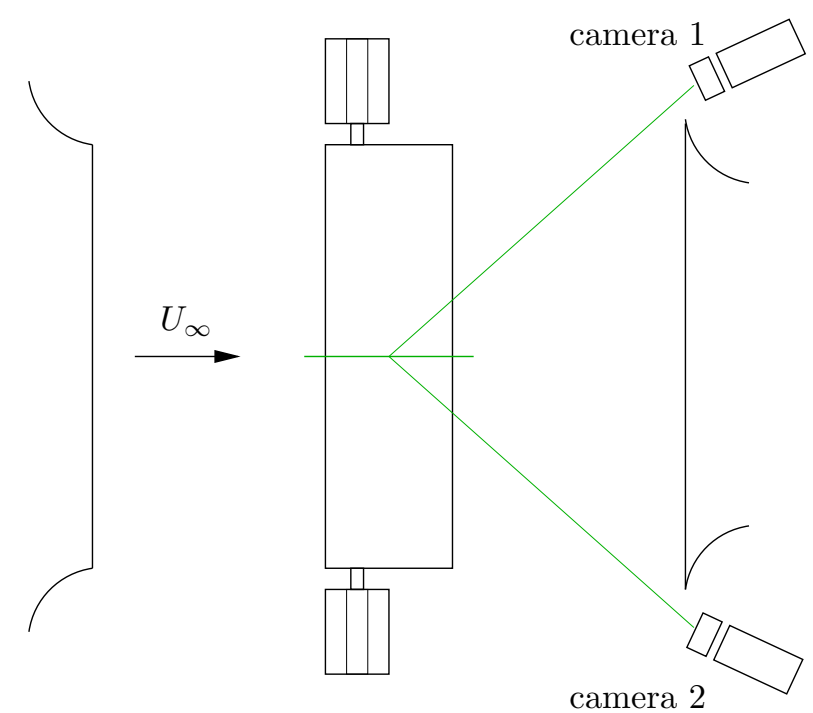

Figure 1. Schematic representation of the PIV set-up in the test section of DLR's low speed wind tunnel.

\section{Identification of Vortical Structures}

The first step towards analysing the unsteady dynamics of the flow is the identification of the dynamically significant, large-scale vortical regions contained in a given flowfield. The main existing vortex indicators can be roughly divided into intuitive measures and methods based on the analysis of the velocity gradient tensor $\nabla \boldsymbol{u}$. The use of common intuitive indicators, comprising local pressure minimum, isovorticity surfaces and closed or spiralling pathlines, might be inefficient or even fail for the detection of vortices in unsteady flows or in flows with a large amount of shear ${ }^{12}$. For this type of flows a vortex identification scheme should be capable of identifying numerous vortices advecting at different speeds, providing Galilean invariance.

Three well known Galilean invariant vortex criteria derived from the velocity gradient tensor are the $Q$-criterion introduced by Hunt et al. ${ }^{16}$, the $\Delta$-criterion of Chong et al. ${ }^{17}$ and the $\lambda$-criterion proposed by Jeong and Hussain ${ }^{11}$. According to the respective definitions vortices are regions where

$$
\begin{gathered}
Q=\frac{1}{2}\left(|\boldsymbol{\Omega}|^{2}-|\boldsymbol{S}|^{2}\right)>0, \\
\Delta=\left(\frac{Q}{3}\right)^{3}+\left(\frac{\operatorname{det} \nabla \boldsymbol{u}}{2}\right)^{2}>0, \\
\lambda_{2}(\boldsymbol{S}+\boldsymbol{\Omega})^{2}<0,
\end{gathered}
$$

where $\boldsymbol{S}=\frac{1}{2}\left[\nabla \boldsymbol{u}+(\nabla \boldsymbol{u})^{T}\right]$ and $\boldsymbol{\Omega}=\frac{1}{2}\left[\nabla \boldsymbol{u}-(\nabla \boldsymbol{u})^{T}\right]$ are the symmetric and antisymmetric components of $\nabla \boldsymbol{u}$ respectively, and $\lambda_{2}$ is the second largest eigenvalue of the tensor $\boldsymbol{S}^{2}+\boldsymbol{\Omega}^{2}$.

However, these methods perform a local analysis of the flow field, while vortical structures are inherently non-local phenomena. Hence, these criteria are not always adapted for the analysis of complex, highly inhomogeneous flows, in particular when structures of various dimensions and strength are present. Furthermore, vortex identification criteria based on $\nabla \boldsymbol{u}$ are inadequate for application on experimental data, such as instantaneous velocity fields measured with PIV, where measurement noise can severely contaminate the derivatives. Another important issue arises when investigating three-dimensional vortical structures in a two-dimensional measurement plane, where the detectability of a vortex is strongly affected when its axis is not perpendicular to the measurement plane. This also applies for the present data where it is assumed that the flow at the model mid-span is fairly symmetric, which permits the application of detection criteria based on the two-dimensional geometry of the vector field.

For a given instantaneous velocity field the possible vortex cores based on the $Q$-, $\Delta$ - and $\lambda_{2}$-definitions (3)-(5) are determined and depicted in figure 2. The $\lambda_{2}$-criterion seems to be the most suitable for this application. Compared to the results of the $Q$ - and $\Delta$-criterion in the leading edge region, the $\lambda_{2}$-criterion 

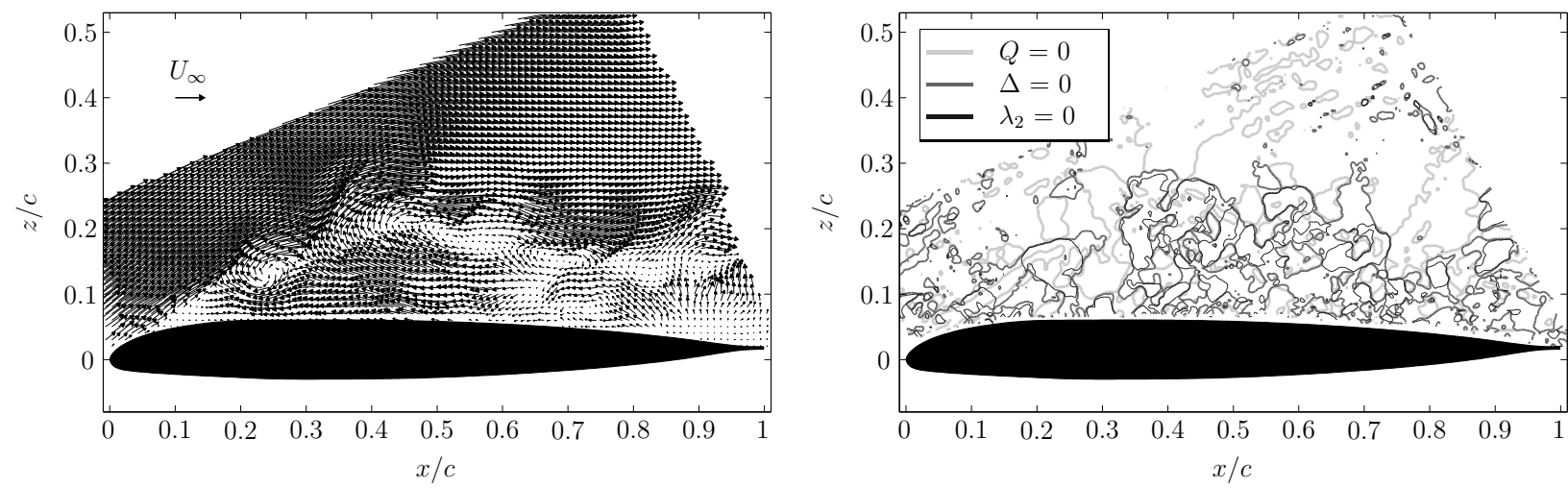

Figure 2. Instantaneous velocity field (left) and corresponding vortex contours determined with the $\nabla u$-derived definitions (right) at $\alpha=25.9^{\circ}$ on the downstroke $\left(\alpha_{0}=18^{\circ}, \alpha_{1}=8^{\circ}\right.$, and $\left.k=0.10\right)$.

is capable of distinghuishing between rotation due to pure shear and due to an actual swirling motion. Nevertheless, none of the criteria allows for the detection of individual vortex cores. Therefore a non-local, Galilean invariant procedure that does not require the computation of derivatives was chosen for this study.

The alternative vortex centre identification algorithm used here is based on a dimensionless scalar function $\Gamma$, derived directly from the velocity field as was first proposed by Michard et al. ${ }^{18}$. The function $\Gamma$ is defined as

$$
\Gamma\left(x_{i}\right)=\frac{1}{N} \sum_{\mathbf{x}_{\mathbf{j}} \in S} \frac{\left[\left(\boldsymbol{x}_{\boldsymbol{j}}-\boldsymbol{x}_{\boldsymbol{i}}\right) \times\left(\boldsymbol{u}_{\boldsymbol{j}}-\tilde{\boldsymbol{u}}_{\boldsymbol{i}}\right)\right] \cdot \boldsymbol{n}}{\left|\boldsymbol{x}_{\boldsymbol{j}}-\boldsymbol{x}_{\boldsymbol{i}}\right| \cdot\left|\boldsymbol{u}_{\boldsymbol{j}}-\tilde{\boldsymbol{u}}_{\boldsymbol{i}}\right|}=\frac{1}{N} \sum_{\mathbf{x}_{\mathbf{j}} \in S} \sin \left(\theta_{i j}\right)
$$

where $S$ is a two-dimensional area around $\boldsymbol{x}_{\boldsymbol{i}}, N$ is the number of points $\boldsymbol{x}_{\boldsymbol{j}}$ inside $S$, and $\boldsymbol{n}$ is the unit vector normal to the plane. The velocity in $\boldsymbol{x}_{\boldsymbol{j}}$ is given by $\boldsymbol{u}_{\boldsymbol{j}}$ and $\tilde{\boldsymbol{u}}_{\boldsymbol{i}}$ denotes the local velocity around $\boldsymbol{x}_{\boldsymbol{i}}$, which is the spatial average over a small surface surrounding $\boldsymbol{x}_{\boldsymbol{i}}$. The local velocity is taken into account in order for $\Gamma$ to be Galilean invariant. The angle $\theta_{i j}$ is the angle formed by the vectors $\boldsymbol{x}_{\boldsymbol{j}}-\boldsymbol{x}_{\boldsymbol{i}}$ and $\boldsymbol{u}_{\boldsymbol{j}}-\tilde{\boldsymbol{u}}_{\boldsymbol{i}}$ as illustrated in figure 3 . According to this definition, $\Gamma$ is a dimensionless scalar function, with $-1 \leq \Gamma \leq 1$, where the

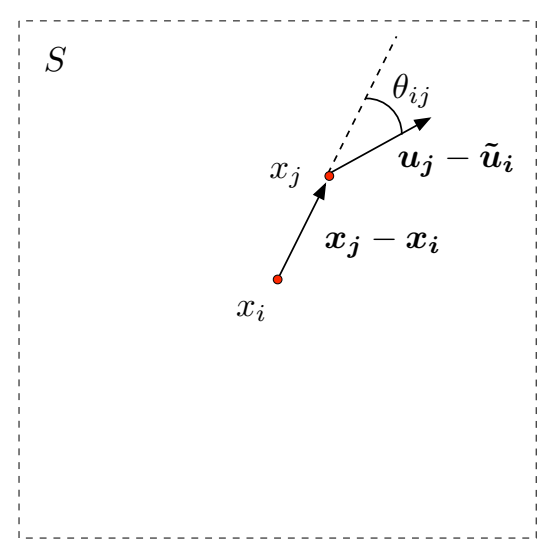

Figure 3. Definition sketch for the computation of $\Gamma$. location of possible vortex cores is determined by the local extrema of $\Gamma$ and the direction of rotation is given by the sign of the local extremum.

The possible vortex centres detected based on the calculation of $\Gamma$ for the instantaneous velocity field of figure 2 are presented in figure 4 together with the vortex cores determined with the $\boldsymbol{\nabla} \boldsymbol{u}$-derived functions. The circles and tringles indicate clockwise respectively counterclockwise rotating vortices. For all detected vortex centres the conditions (3)-(5) are fulfilled, which demonstrates the effectiveness of the algorithm. The remaining step towards investigation of the spatiotemporal evolution of the vortices is to trace their trajectories over a time series of flow fields, herefore the velocity of the vortex centre within the reference frame has been used to predict its future position and narrow the number of possible follow-up vortices.

\section{Results and Discussion}

Due to the combination of global time resolved imaging and the vortex detection algrithm presented in the previous section, the characteristic features of the dynamic stall process can be examined within a single oscillation. With view to the present data, the unsteady flow development over an oscillation airfoil was divided into different stages analogously to the classification of Shih et al. ${ }^{5}$ with regard to the flow over an airfoil pitching-up at constant rate. A general description of the dynamic phenomena associated with the different stages is attempted based on a sequence of instantaneous velocity and vorticity fields and the corresponding airfoil surface pressure distributions. It will be focused on the separation mechanism, the time scales associated with the different stages prior to stall, and the influence of unsteadiness on stall delay. 

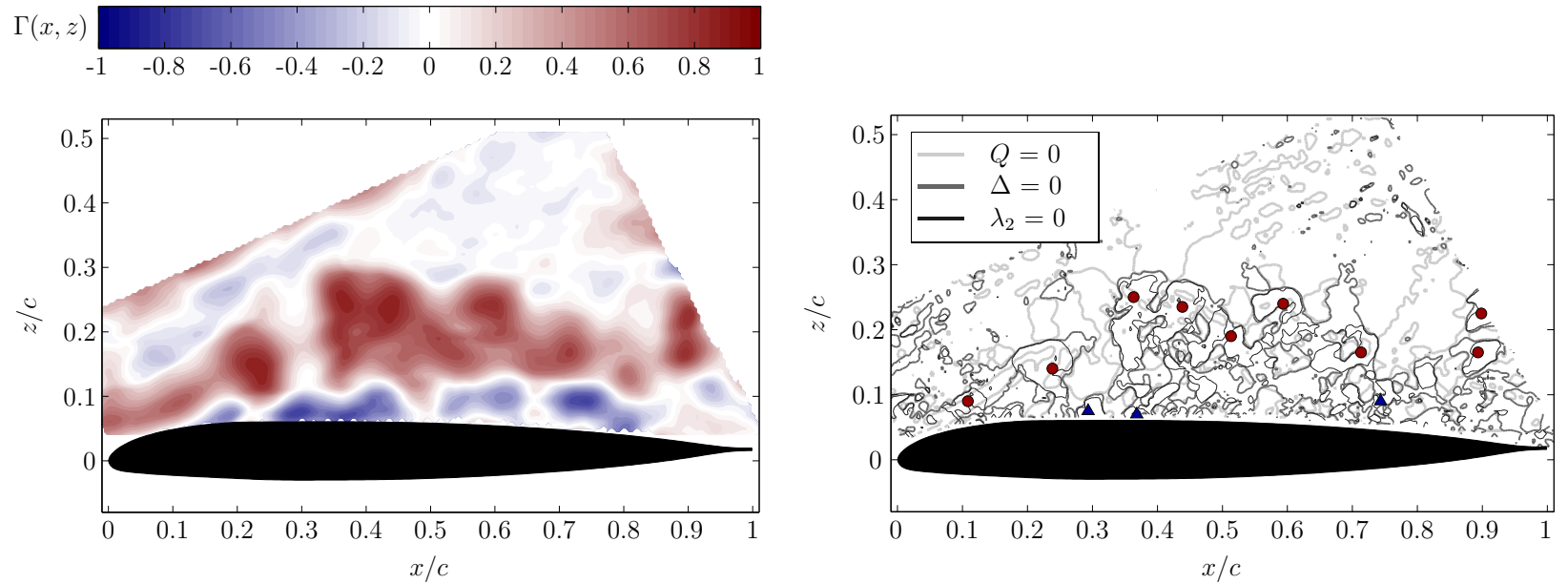

Figure 4. Location of clockwise $(\bullet)$ and counterclockwise rotating $(\Delta)$ vortex cores (right) based on the calculation of $\Gamma$ (left) for the instantaneous velocity field of figure $2\left(\alpha=25.9^{\circ}\right.$ on the downstroke for $\alpha_{0}=18^{\circ}, \alpha_{1}=8^{\circ}$, and $\left.k=0.10\right)$.

\section{IV.A. Dynamic Separation}

The image sequence in figure 5 presents the prominent features of the dynamic stall life cycle. All images were taken from within a single oscillation with $\alpha_{0}=20^{\circ}$, with $\alpha_{1}=8^{\circ}$ and $k=0.10$. The dimensionless out of plane component of the vorticity is colour-coded and only every fifth velocity vector is shown for the sake of visibility. The position of vortices, as determined by the detection algorithm described in section III, are indicated by circles for clockwise rotating vortices and triangles for the counterclockwise rotating ones. The associated evolution of lift coefficient $\left(C_{l}\right)$ and pitching moment coefficient $\left(C_{m}\right)$ is depicted in figure 6 . The static air loads are presented by the gray lines, which reveal a static stall angle of attack $\alpha_{\mathrm{ss}}=21.4^{\circ}$. The markers correspond to the snapshots in figure 5.

During the upstroke motion, until the airfoil reaches its static stall angle, the flow is attached to the surface and there is a strong leading edge suction peak near the leading edge (figure 5(a)). When the upstroke motion continues beyond the static stall angle, the adverse pressure gradient on the suction side increases, causing flow reversal to develop from the trailing edge up towards the leading edge region (figure 5(b)-5(c)). Clockwise rotating vortical structures develop in the shear layer at the interface of the reversing flow and the external flow. Although the reversing flow layer extends along almost the entire chord, the boundary layer stays close to the surface and the vortices are not ejected into the external flow. Due to the local thickening of the boundary layer at the leading edge the flow is accelerated around the nose causing the suction peak to grow. As a consequence the lift, since integrated from the pressure distribution, keeps increasing as well but with a slightly different slope than below $\alpha_{\mathrm{ss}}$. The latter is due to the fact that the suction at the trailing edge neutralizes when the flow separates. This differs significantly from the flow over a stationary airfoil, where flow reversal implies departure of the boundary layer from the airfoil's surface, leading to a dramatic decrease of the lift and pitching moment.

The shear layer vortices in figure 5(c) are relatively small, of the order of the shear layer thickness. With increasing time the Kelvin-Helmholtz type of instability of the shear layer grows, leading to the development of larger individual vortical structures (figure $5(\mathrm{~d})$ ). These individual clockwise rotating vortices evolve very quickly and tend to merge into much larger structures. This is clearly visible in figure $5(\mathrm{e})$ where multiple vortices seem to roll up into a large dynamic stall vortex, resulting in a plateau in the surface pressure distribution between approximately $0.2 c$ and $0.5 c$ on the suction side. The primary large-scale coherent structures, referred to as dynamic stall vortices, consist of a combination of the rolled-up shear layer and the remnants of several vortices generated by the shear layer instability. The part of the dynamic stall cycle that starts with the onset of flow reversal and decribes the formation of the primary stall vortex is referred to as the vortex formation stage.

Strong inviscid/viscous interactions between the stall vortices and the reversing flow layer near the airfoil's surface lead to the emergence of counter-rotating vortices that seem to push the primary vortices away from the surface. Analogue to the formation process of vortices in the wake of a cylinder ${ }^{19}$, this primary stall vortex grows to saturation before being pinched off from the separated boundary layer that provides its circulation 

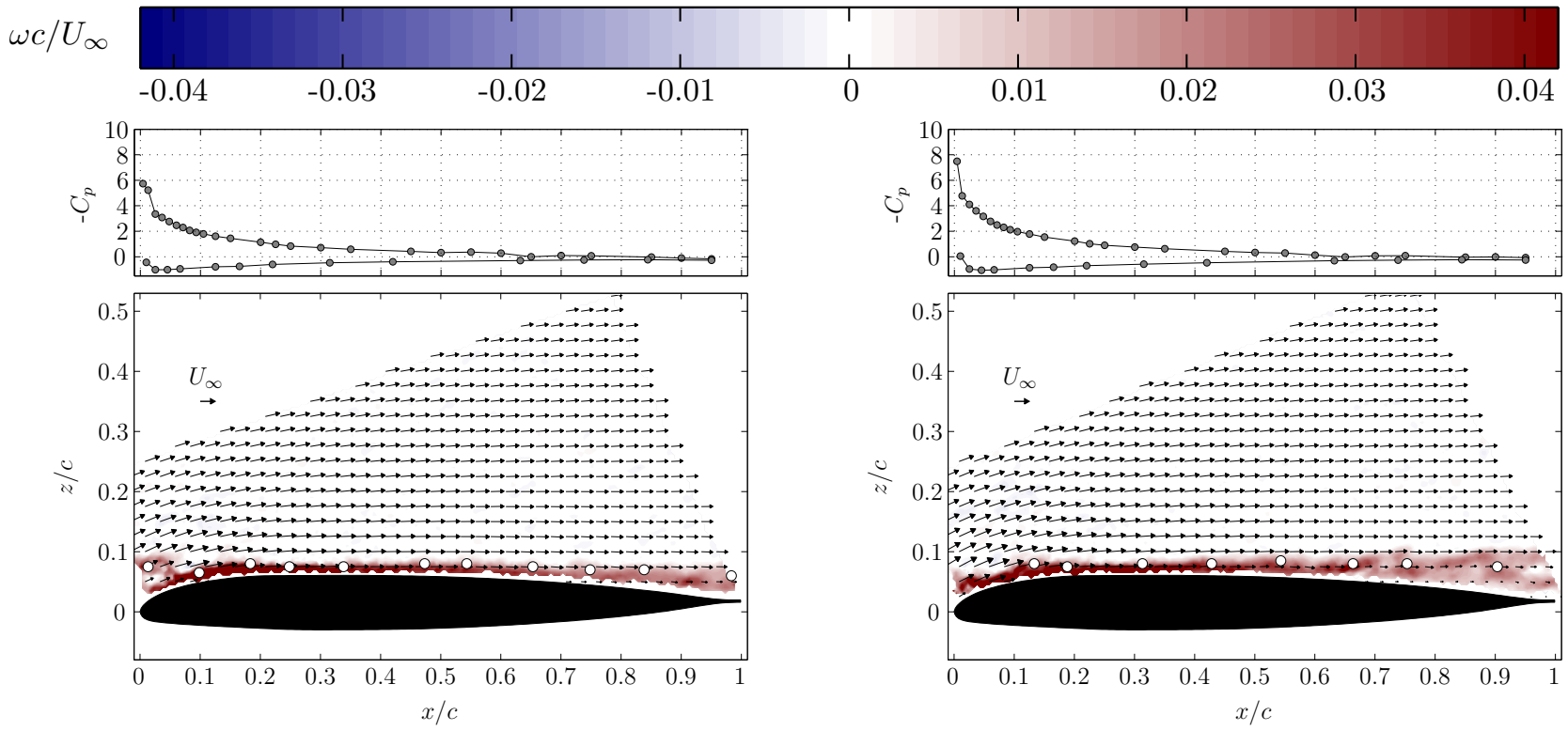

(a) $\alpha=21.5^{\circ}$ on the upstroke

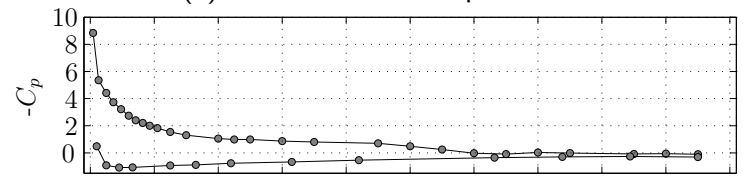

(b) $\alpha=24.5^{\circ}$ on the upstroke

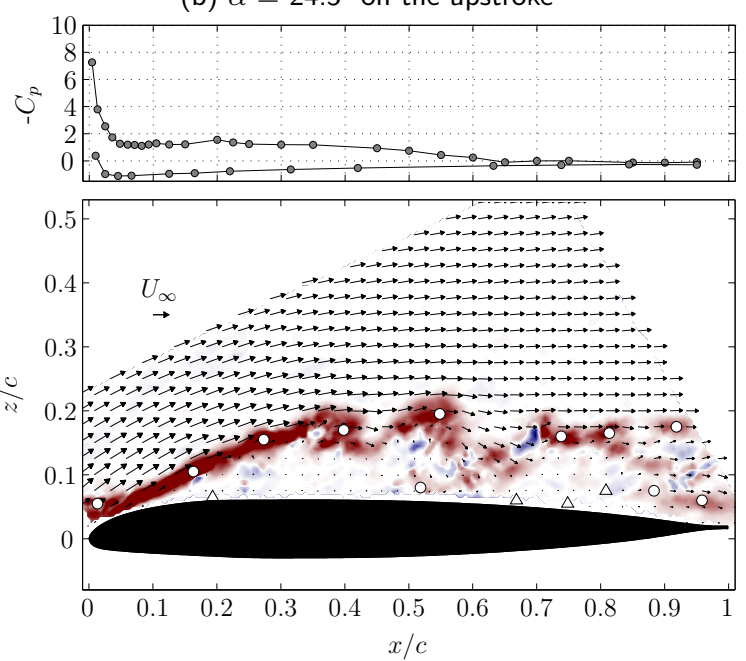

(c) $\alpha=26.8^{\circ}$ on the upstroke
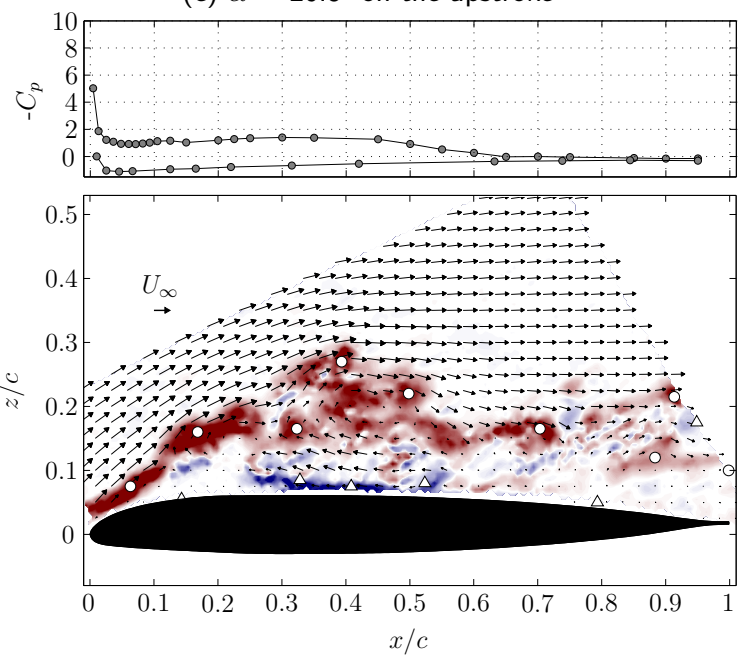

(e) $\alpha=27.9^{\circ}$ on the upstroke

(d) $\alpha=27.6^{\circ}$ on the upstroke
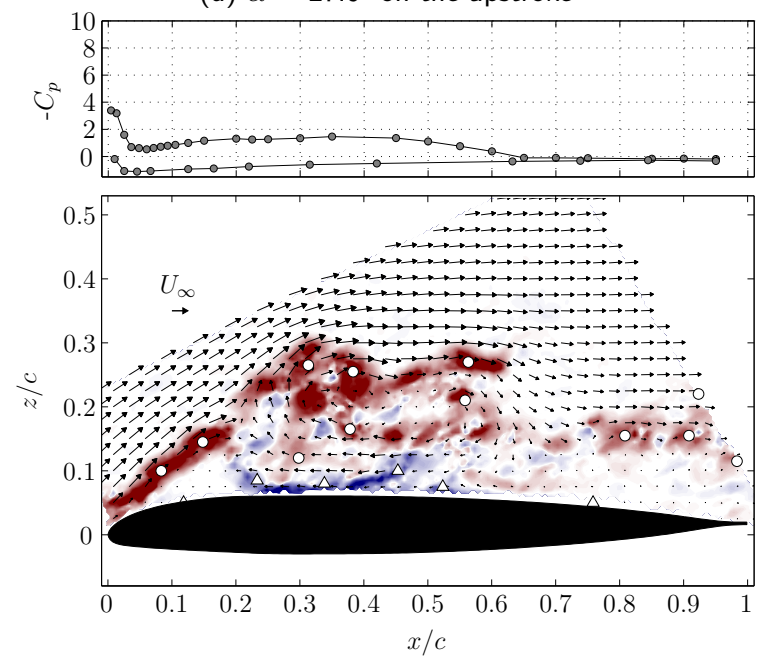

(f) $\alpha=27.9^{\circ}$ on the upstroke 


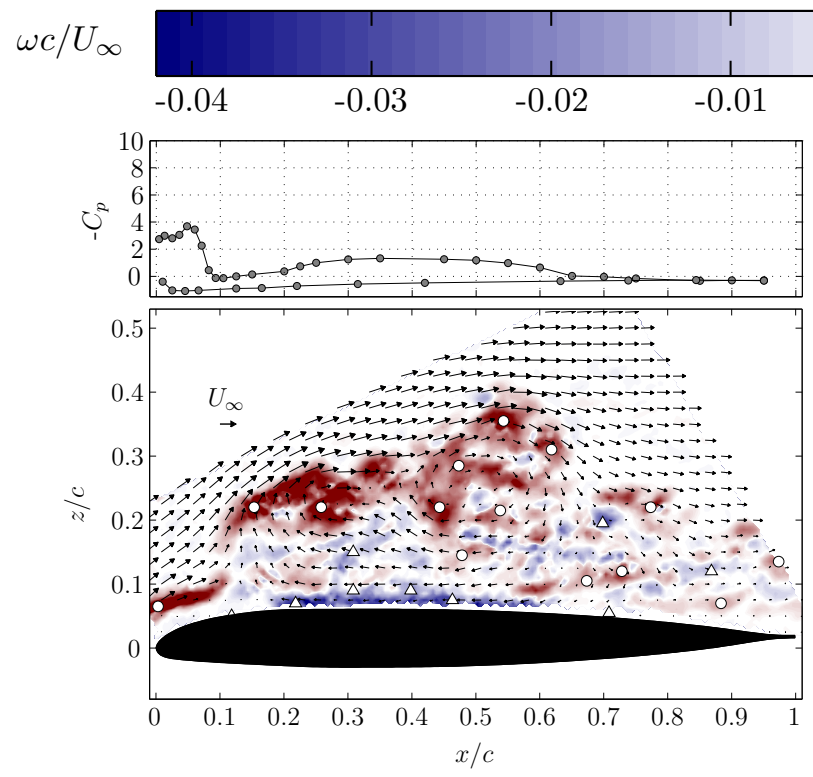

(g) $\alpha=28.0^{\circ}$ on the upstroke

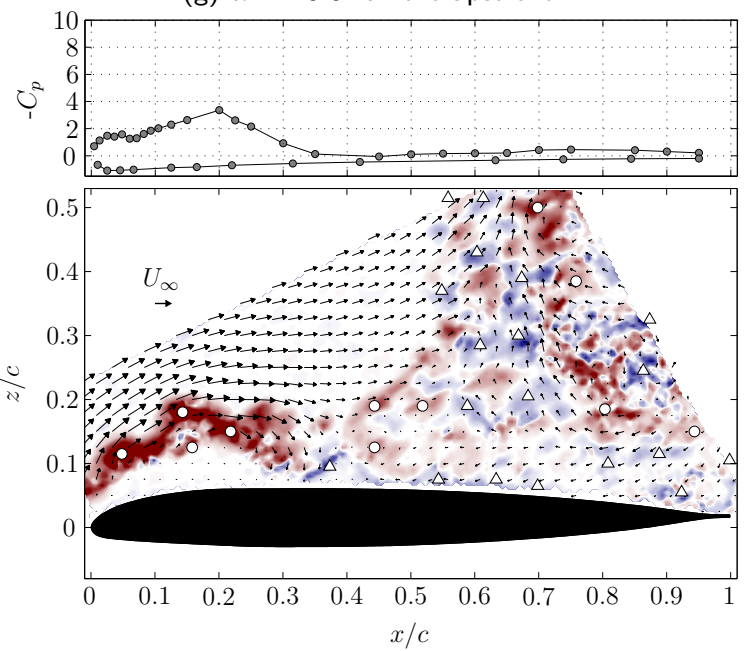

(i) $\alpha=27.9^{\circ}$ on the downstroke

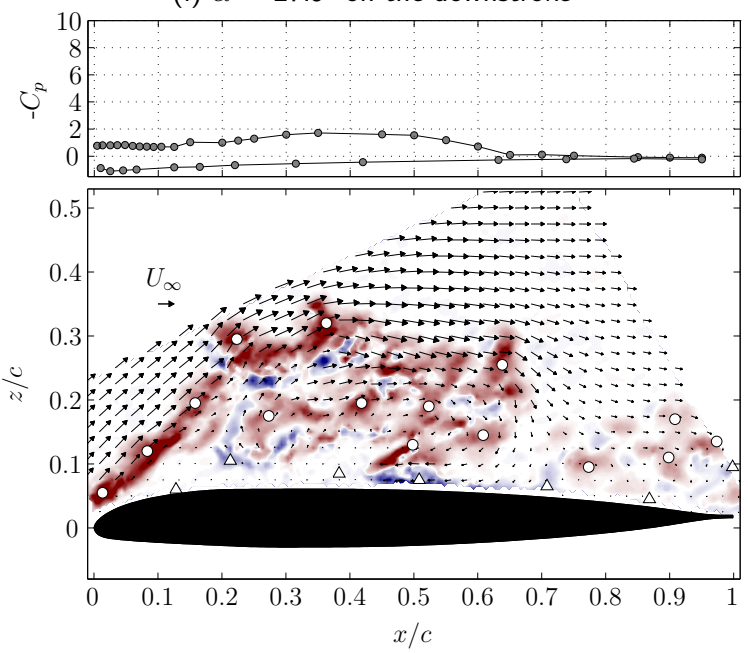

(k) $\alpha=27.5^{\circ}$ on the downstroke
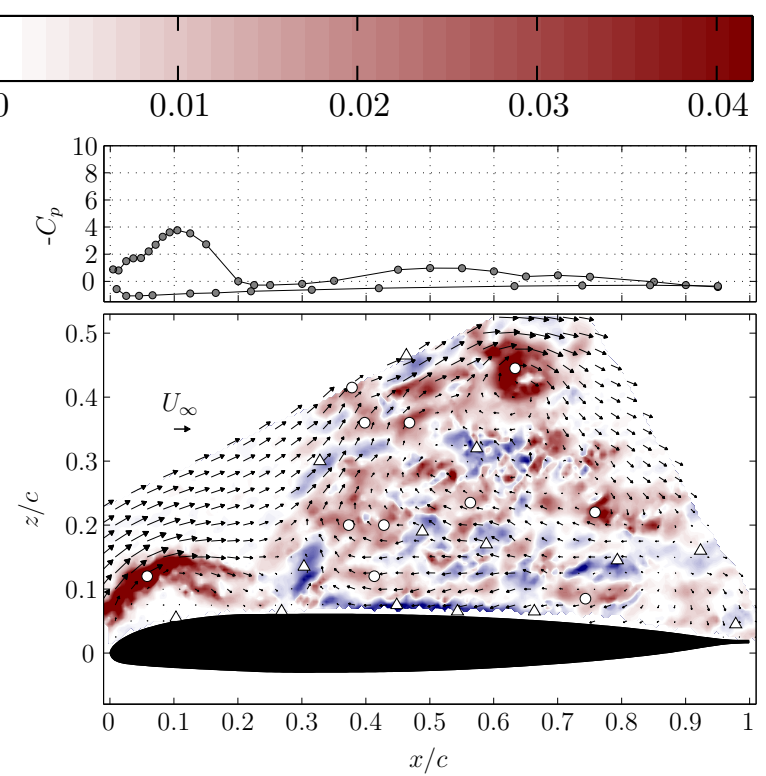

(h) $\alpha=28.0^{\circ}$ on the downstroke

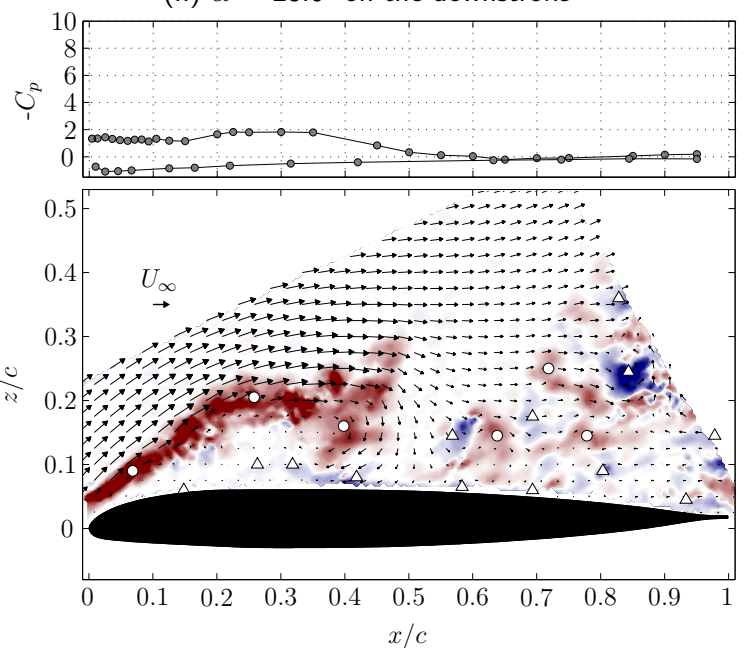

(j) $\alpha=27.7^{\circ}$ on the downstroke
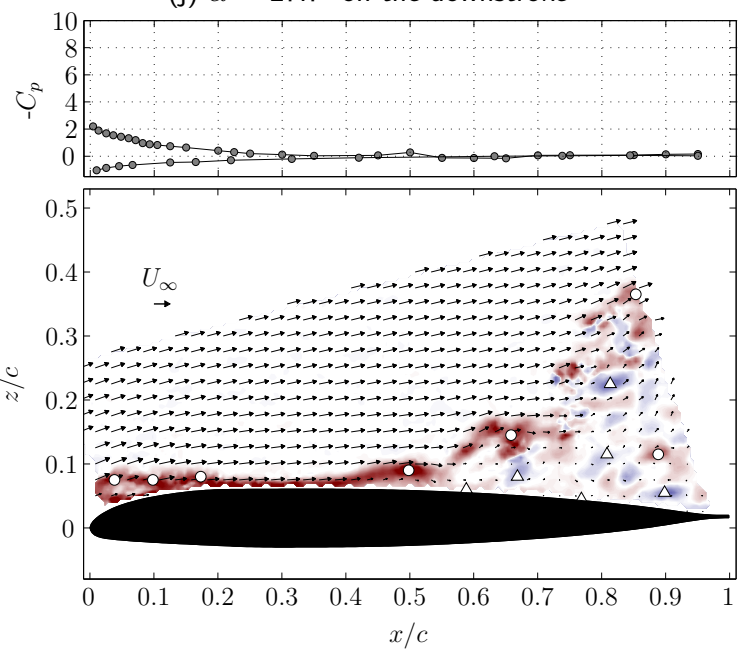

(I) $\alpha=16.5^{\circ}$ on the downstroke

Figure 5. Instantaneous flow fields with detected vortex cores $(\bigcirc$ : clockwise, $\triangle$ : counterclockwise rotating) and the respective surface pressure distribution for the states marked in figure 6 within a single oscillation $\left(\alpha_{0}=20^{\circ}, \alpha_{1}=8^{\circ}\right.$, and $k=0.10)$. 

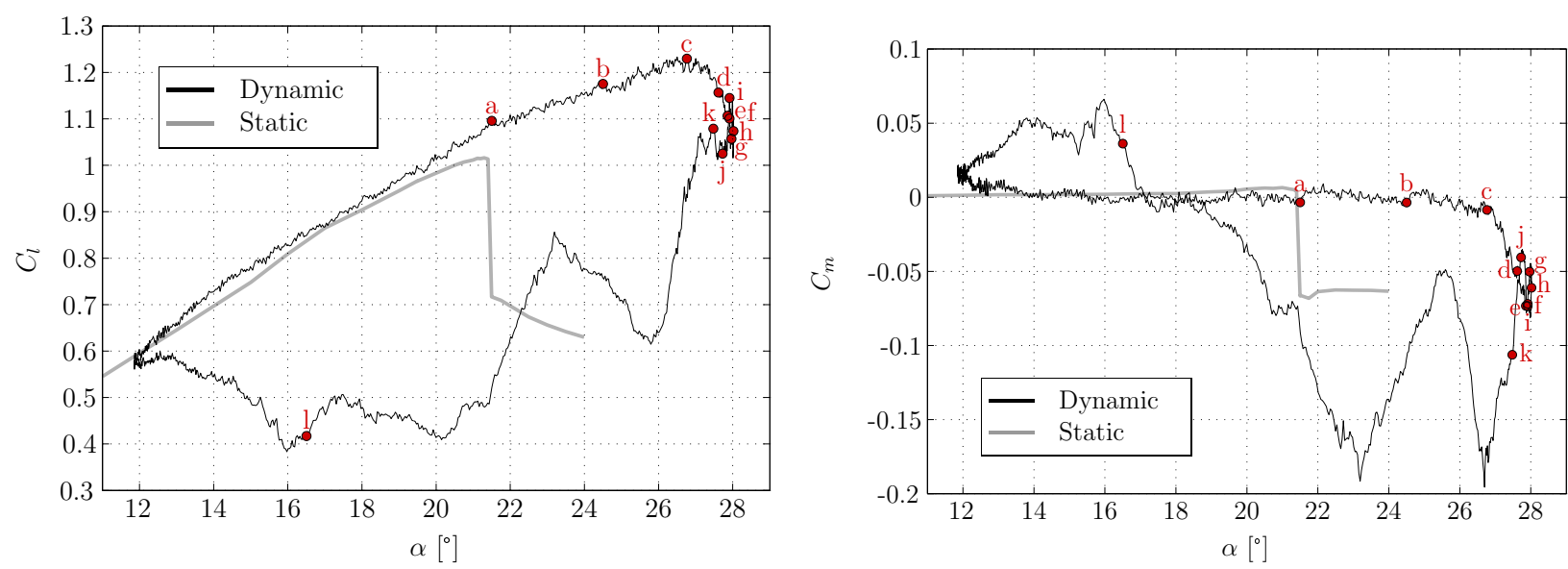

Figure 6. Lift (left) and pitching moment (right) coefficient hysteresis for a single oscillation $\left(\alpha_{0}=20^{\circ}, \alpha_{1}=8^{\circ}\right.$, and $k=0.10)$ together with their static counterparts (-).

(figure 5(f)). The additional circulation accumulates in the shear layer leading to the formation of a secondary stall vortex. Both separated leading edge structures are convected downstream. As a consequence the flow can follow the nose again (figure $5(\mathrm{~g})$ ), which in turn gives rise to the increased suction near the leading edge seen in the pressure distribution. The resulting massive separation starting at approximately $0.25 c$ slows down the convection of vorticity from the leading edge forcing the leading edge shear layer to roll up such as a separation bubble is formed (figure $5(\mathrm{~h})$ ). During downstroke the separation bubble grows and moves slowly downstream as can be recognised both in the surface pressure distributions and the velocity fields in figure $5(\mathrm{~h})-5(\mathrm{k})$. When this large coherent structure is convected downstream, it will cause the boundary layer at the leading edge to reattach. Flow reversal redevelops in form of a separation bubble as long as the adverse pressure gradient near the airfoil's surface is dominant, which then leads to a break down of the shortly reattached flow.

This alternation of boundary layer separation and reattachment, which causes the large fluctuations of the air loads during the downstroke motion, continues until the adverse pressure gradient at the leading edge is to weak or disappears at all and no reversing flow and subsequent large-scale coherent structures are formed (figure 5(1)).

\section{IV.B. Dynamic Stall Delay}

In the absence of dynamic effects, the flow over an airfoil is massively separated for $\alpha$ exceeding the static stall angle, whereas massive flow separation associated with dynamic stall may be delayed to much higher angles of attack. The actual dynamic stall delay is defined as the delay between the time the effective angle exceeds the static stall angle $\left(t_{\alpha_{\mathrm{ss}}}\right)$ and onset of dynamic stall $\left(t_{\alpha_{\mathrm{ds}}}\right)$, which is marked by the detachment of the dynamic stall vortex from the boundary layer. The attempt to assess the stall delay from the measured velocity fields is based on the observation of the fact that prior to stall during the vortex formation stage the vortical structures that are formed in the shear layer stay close to the surface and are not ejected into the external flow. However, the average distance of the shear layer, respectively shear layer vortices from the airfoil's upper surface increases until the small-scale structures are subjected to a higher order instability and tend to merge into the dynamic stall vortex. Therefore the average distance $(\Delta z)$ of the experimentally determined vortical structures normal to the surface was calculated for fixed phase angles and is presented in figure 7 as a function of geometrical angle of attack and time.

In the first part of the upstroke motion the flow is fully attached and no vortical structures should be present. The scatter in this region is due to experimental limitations near the airfoil's surface and the malfunction of the vortex detection algorithm here. After passing beyond the static stall angle the detected coherent structures represent the shear layer vortices and their average normal vertical displacement $(\Delta z)$ is equivalent to the average height of the shear layer. Initially the average height increases slightly and approximately linearly up to a certain point where the shear layer rolls up into the dynamic stall vortex, which is then pinched off from the boundary layer and ejected into the external flow. This latter stage is referred to as the vortex convection stage. The close-up view in figure 8 exhibits a linear increase of the 

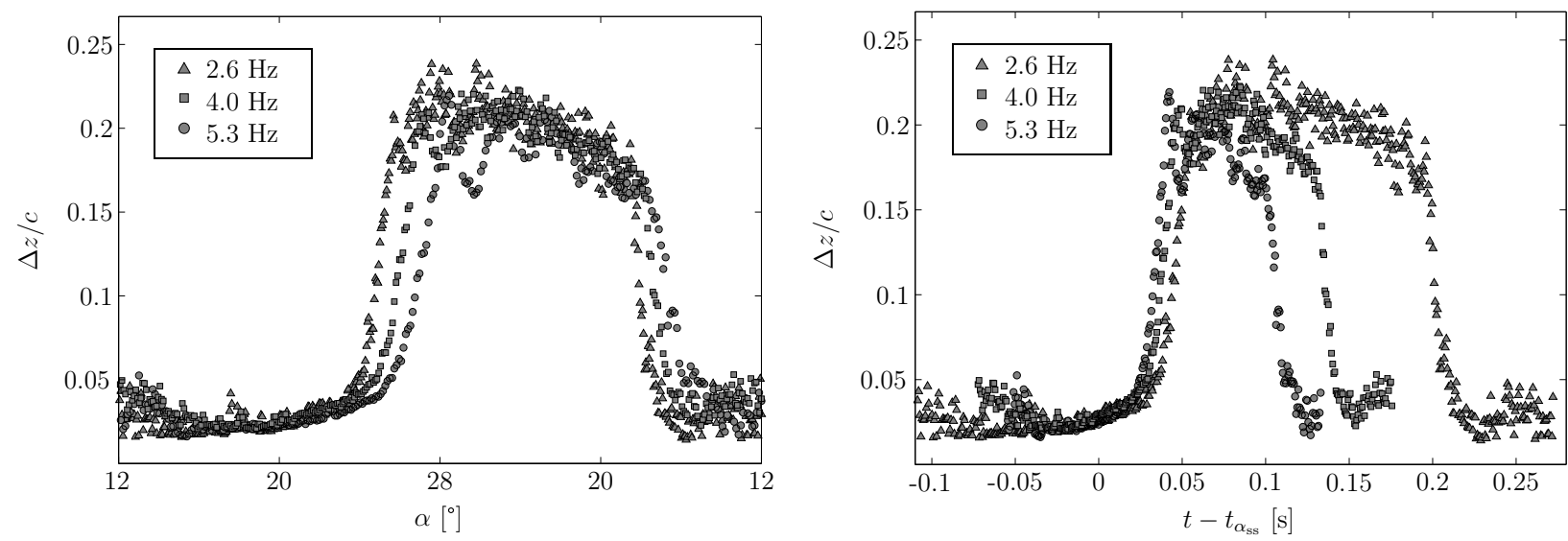

Figure 7. Average vertical distance of the vortex cores from the airfoil's upper surface versus angle of attack (left) and versus time lag with respect to $t_{\alpha_{S S}}$, the time at which the angle of attack passes beyond the static stall angle (right), for different degrees of unsteadiness and $\alpha_{0}=20^{\circ}, \alpha_{1}=8^{\circ}$.

vertical displacement during the vortex convection stage. The slope of the fitting curve represents the vertical component of the vortex convection speed. The transition of the vortex formation stage into the convection stage corresponds to stall onset. Hence dynamic stall onset has been assessed from the intersection of the two linear fitting curves.

In the right part of figure 7 the curves are shifted such that the zero on the abscissa coincides with the time the effective angle exceeds the static stall angle $\left(t_{\alpha_{\mathrm{ss}}}\right)$. The effective angle is the angle between the airfoil's chord and the relative wind. When dynamic effects are present the effective wind is found by substracting the motion induced velocity at the leading edge from the external flow. The influence on the magnitude is less than $0.3 \%$ of $U_{\infty}$ and it is therefore neglegible. The influence on the effective angle of attack $\alpha_{\text {eff }}$ is taken into account for the calculation of the stall delay.

For all cases in figure 7, corresponding to different oscillation frequencies, i.e. different degrees of unsteadiness, the mean angle of attack and amplitude are $\alpha_{0}=20^{\circ}$ and $\alpha_{1}=8^{\circ}$ allowing for the isolated influence of the reduced frequency to be examined. With regard to angle of attack, stall is further delayed with increasing frequency, whereas with regard to the actual time delay, the onset of stall is sooner for larger oscillation frequencies. As already pointed out by Currier and Fung ${ }^{20}$, unsteadiness actually speeds up the separation process rather than delaying it. Visbal and Shang ${ }^{21}$ also report a delay of stall to larger angle of attack for higher pitch rates. Additionally, they studied numerically the influence of the pitch-axis location. They suggested using the velocity of the nose as a measure of the stage of evolution of the flow. Following this suggestion, the stall delay is presented in figure 9 as a function of the magnitude of the motion induced velocity of the airfoil at the leading edge $v_{\mathrm{le}}$ at $t=t_{\mathrm{ss}}$. For the present data a tendency towards shorter delays for increasing nose speeds is found.

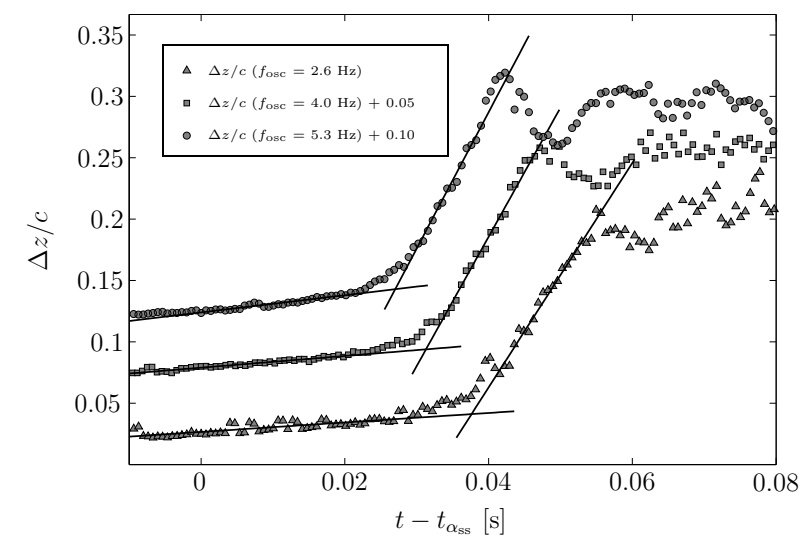

Figure 8. Detail of figure 7 (right) with the corresponding linear fits. 

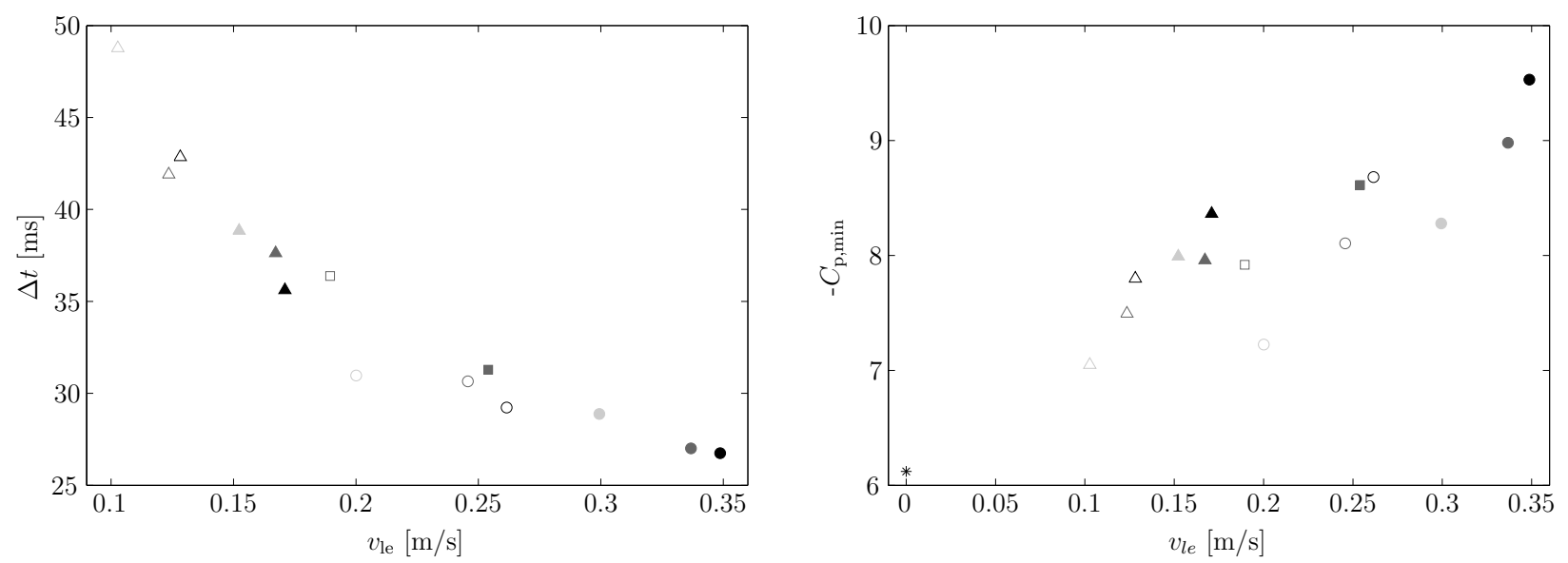

Figure 9. Stall delay (left) and minimum surface pressure level (right) versus the motion induced speed of the airfoil's leading edge for different oscillation parameter combinations: $f_{o s c}=2.5 \mathrm{~Hz}(\Lambda), 4.0 \mathrm{~Hz}(\bullet), 5.3 \mathrm{~Hz}(\bullet), \alpha_{0}=18^{\circ}(\triangle)$, $20^{\circ}(\triangle), 2^{\circ}(\triangle), \alpha_{1}=6^{\circ}(\triangle), 8^{\circ}(\triangle)$ and the static case $(*)$.

The calculated values of the dynamical stall angle, the stall delay and the leading edge speed as well as the minimum surface pressure level or suction peak $C_{\mathrm{p}, \mathrm{min}}$, are listed in table 1. Figure 9 depicts the relation between the suction peak and the leading edge speed. The scatter displays a tendancy toward strong suction when the nose speed is increased. The data point corresponding to the static case has been included as well and supports the tendency. This observation provides a hint towards the mechanisms leading to stall onset. The suction peaks are an indication of the strength of the leading edge vortex. ${ }^{21} \mathrm{~A}$ stronger vortex implies stronger inviscid/viscous interaction between the vortex and the reversing flow layer that separates it from the airfoil's surface. As a consequence the shear layer induced counterrotating vortices will be stronger and they will react in a way that they push the primary vortex away from the surface. A larger nose speed implies a stronger stall vortex that can more easily break away from the boundary layer, leading to a shorter stall delay.

For the range of oscillating motion presented in the study, the motion induced speed of the leading edge at static stall angle appears to be the appropriate parameter to describe the unsteadiness with respect to stall delay. A more systematic study, including an investigation of the Reynolds number effects would be necessary to sustain the previous assumption and to understand the physical key mechanism.

Furthermore, for the present data, the boundary layer separation in both cases, static and dynamic, is

Table 1. Overview of the assessed dynamic stall parameters for different oscillation parameter combinations.

\begin{tabular}{ccccccc}
$f_{\text {osc }}[\mathrm{Hz}]$ & $\alpha_{0}\left[^{\circ}\right]$ & $\alpha_{1}\left[^{\circ}\right]$ & $\alpha_{d s}\left[^{\circ}\right]$ & $\Delta t[\mathrm{~ms}]$ & $v_{\text {le }}\left(t_{\mathrm{ss}}\right)[\mathrm{m} / \mathrm{s}]$ & $-C_{\mathrm{p}, \min }$ \\
\hline 2.6 & 18 & 6 & 24.0 & 49 & 0.10 & 7.05 \\
5.3 & 18 & 6 & 24.0 & 31 & 0.20 & 7.22 \\
2.6 & 18 & 8 & 25.2 & 39 & 0.15 & 7.99 \\
5.3 & 18 & 8 & 26.0 & 29 & 0.30 & 8.28 \\
2.6 & 20 & 6 & 25.0 & 42 & 0.12 & 7.49 \\
4.0 & 20 & 6 & 25.6 & 36 & 0.19 & 7.92 \\
5.3 & 20 & 6 & 25.8 & 31 & 0.25 & 8.10 \\
2.6 & 20 & 8 & 25.9 & 38 & 0.17 & 7.96 \\
4.0 & 20 & 8 & 26.9 & 31 & 0.25 & 8.61 \\
5.3 & 20 & 8 & 27.3 & 27 & 0.34 & 8.98 \\
2.6 & 22 & 6 & 25.6 & 43 & 0.13 & 7.80 \\
5.3 & 22 & 6 & 26.8 & 29 & 0.26 & 8.68 \\
2.6 & 22 & 8 & 26.1 & 36 & 0.17 & 8.36 \\
5.3 & 22 & 8 & 28.1 & 28 & 0.35 & 9.35
\end{tabular}



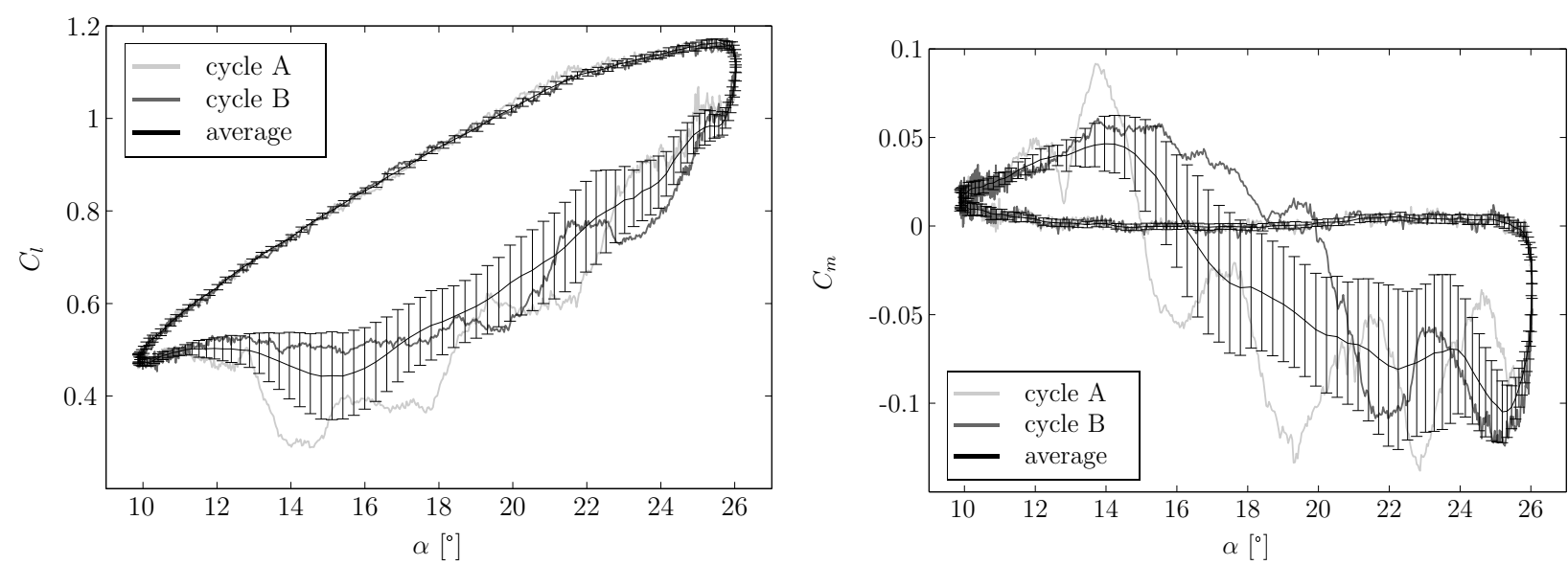

Figure 10. Lift (right) and pitching moment (left) hysteresis for two arbitrary oscillations as well as the average of all recorded cycles where the error bars indicate the standard deviation $\left(\alpha_{0}=18^{\circ}, \alpha_{1}=8^{\circ}\right.$, and $\left.k=0.10\right)$.

initiated by a local adverse pressure gradient. Assuming that the angle of attack at which the local pressure gradient occurs is unaffected by dynamic effects, and solely determined by the external flow characteristics, the airfoil's geometry, and its effective angle of attack, unsteady separation is triggered when the static stall angle is reached. Using this argumentation the dynamic stall delay would be specified by the duration of the formation process of the primary stall vortex.

\section{IV.C. Cycle to Cycle Variations}

In figure 10 the measured air loads, lift and pitching moment, corresponding to different cycles of the forced oscillation around $\alpha_{0}=18^{\circ}$, with amplitude $\alpha_{1}=8^{\circ}$ and reduced frequency $k=0.10$, are presented as well as the cycle average hysteresis curve. The evolution of the aerodynamic coefficients during downstroke displays strong discrepancies between the two arbitrary oscillation periods. In general the reproducibility during the downward pitching part of the cycle is rather poor, due to the intristic characteristics of the dynamic separation process and the sensitivity of the separated flow to variations of the boundary conditions inherent to the experimental process. However, the inconsistency between the two cycles presented here, seems to have different origin. The different evolution of the air loads seems to be a result of different separation behaviour. This becomes more obvious when examining the temporal evolution of the individual pressure signals associated with three pressure sensors closest to the leading edge on the airfoil's suction side $(\mathrm{x} / \mathrm{c}=0.0045,0.0130,0.0250)$ in figure $11(\mathrm{a})$ and figure $11(\mathrm{~b})$. The corresponding integrated air loads are presented in figures $11(\mathrm{c})-11(\mathrm{f})$.

At the beginning of the first downstroke the leading edge suction collapses entirely whereas the signal respective the sensor closest to the leading edge (at $0.45 \%$ chord length from the leading edge) is more elevated during the complete downstroke part of the subsequent cycle. This indicates that there is less flow separation and the flow can still follow the nose contour to a certain extend. This is also evidenced by the discrepancies in the trajectories of the vortices shown in figure 12, where the trajectories of the clockwise rotating vortices emerging during the last quarter of the upstroke and the first two quarters of the downstroke part of the motion are plotted for the different cycles. The evolution of the average vertical displacement of the vortical structures for these particular oscillation periods is presented in figure 13.

Near the end of the upstroke part the boundary layer is thickened and the vertical position of the vortices increases slightly, but they still follow the airfoil contour. After the maximum angle of attack is attained and the downward motion is set in, the structures move further away from the airfoil's surface, but there is a clear difference between the path along which vortices are being convected downstream. This different behaviour can be explained based on the concept of stall delay and formation time introduced earlier. The assessed dynamic stall angle for this particular case equals $\alpha_{\mathrm{ds}}=26.0$ which coincides with the maximum angle of attack or the reversal point of the oscillation. Hence this appears to be a particular case where small perturbation decide whether stall is initiated at the end of the upstroke or at early downstroke, resulting in a different separation behaviour.

The ejection of the primary structure into the external flow in figure $12(\mathrm{c})$ seems to take place on the 


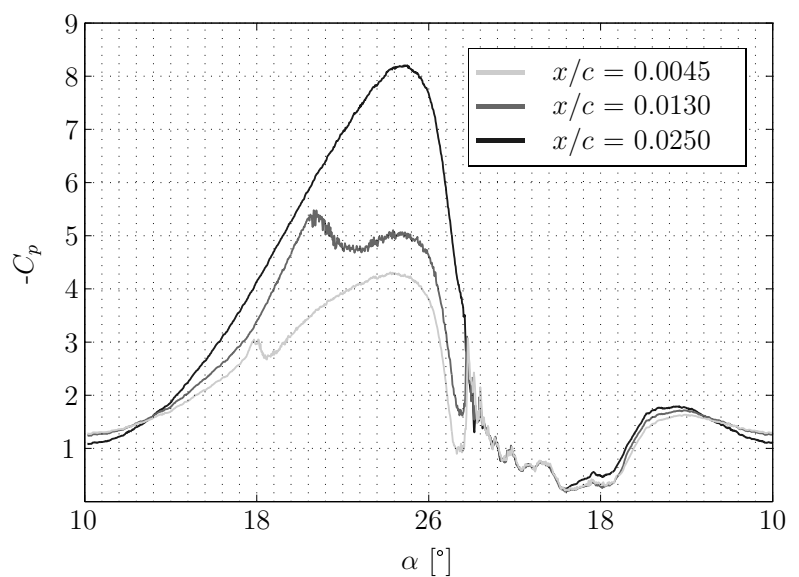

(a)

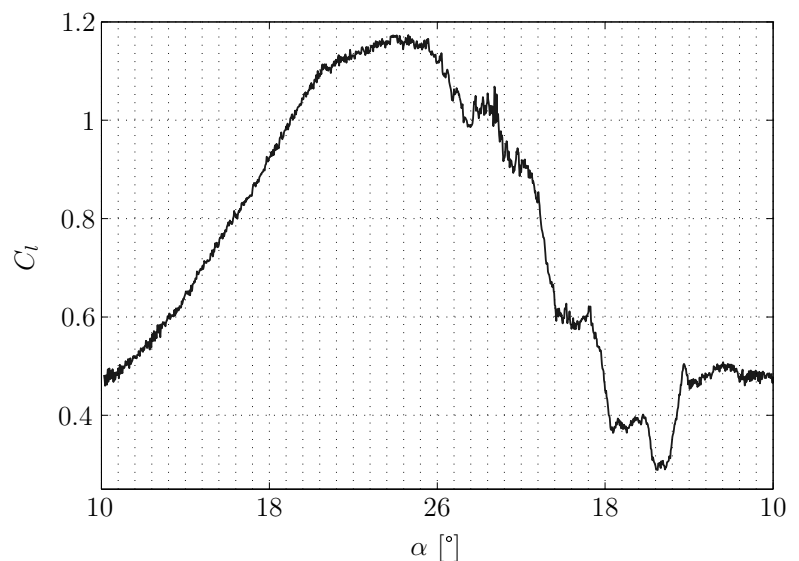

(c)

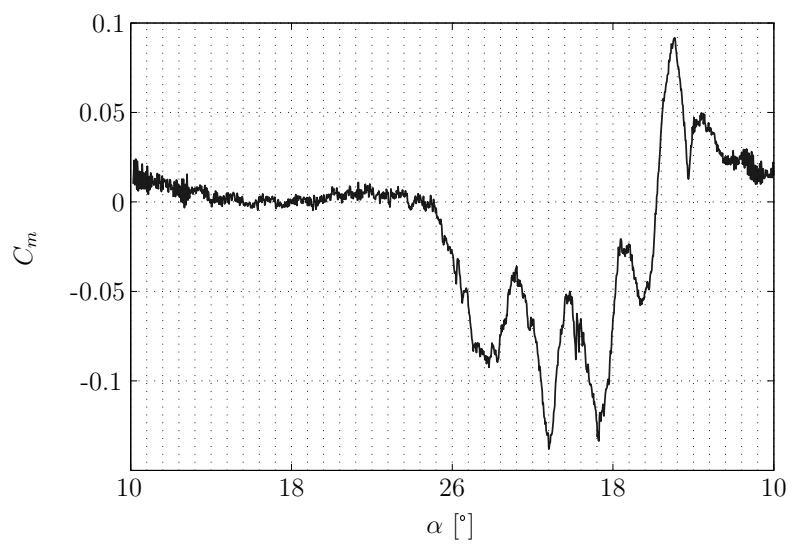

(e)

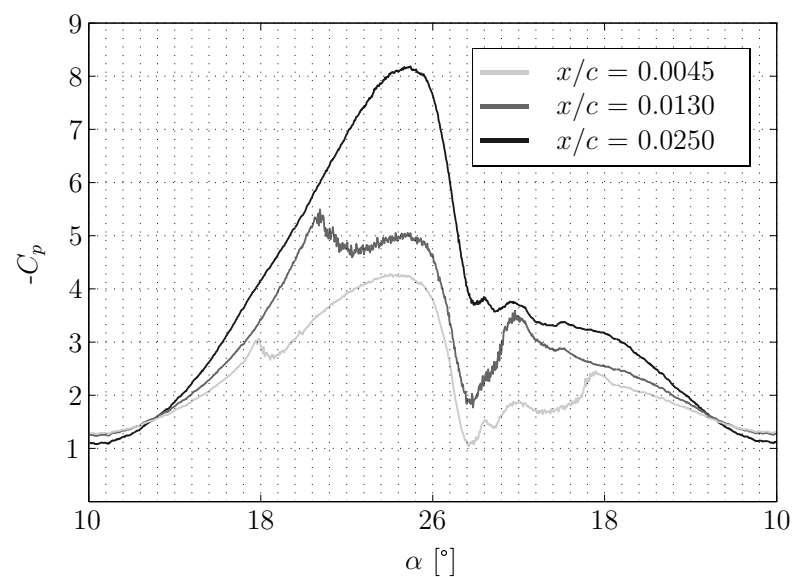

(b)

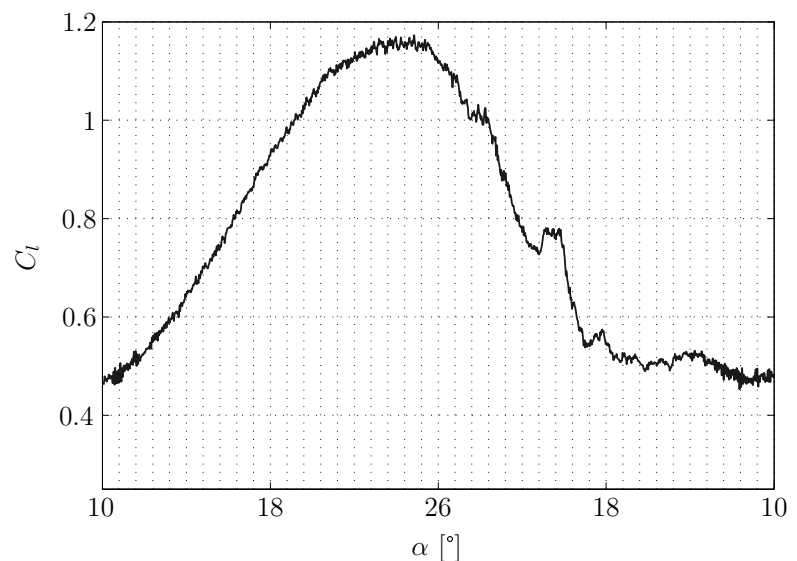

(d)

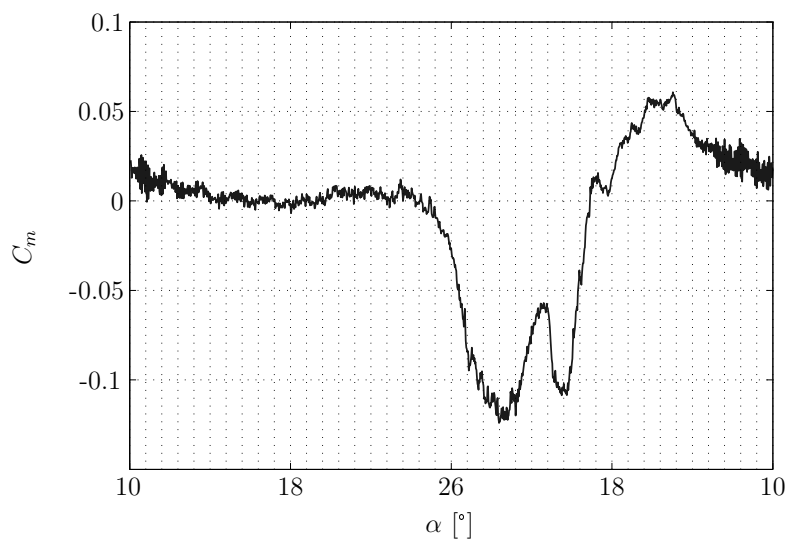

(f)

Figure 11. Temporal evolution of the surface pressure signals generated at the very leading edge on the airfoil's suction side $(\mathrm{a}, \mathrm{b})$, the lift coefficient $(\mathrm{c}, \mathrm{d})$, and the pitching moment coefficient $(\mathrm{e}, \mathrm{f})$ for two arbitrary oscillation cycles A (left) and $\mathrm{B}$ (right) and $\alpha_{0}=18^{\circ}, \alpha_{1}=8^{\circ}, k=0.10$.

upstroke, under an angle larger than the stall angle. Whereas the dynamic stall vortex in the second cycle appears to break away from the boundary layer immediately after the pitching direction reversed, therefore experiencing a downward induced force by the airfoil's leading edge. This could be a reason why the vortex trajectories in figure 12(d) are more gently inclined and yield a smaller separation region and less deflection of the external flow, especially near the leading edge. As a consequence the vorticity formed at the leading edge can be convected downstream and does not accumulate into large coherent structures. Hence, large 


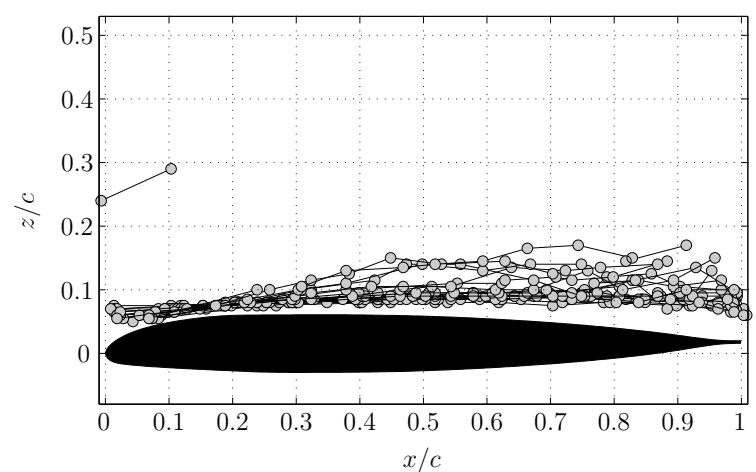

(a) $22^{\circ}<\alpha \leq 26^{\circ}$ on the upstroke

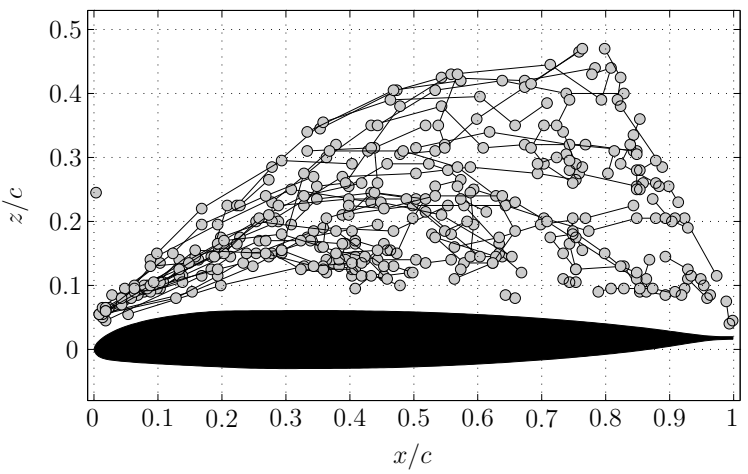

(c) $26^{\circ}>\alpha \geq 22^{\circ}$ on the downstroke

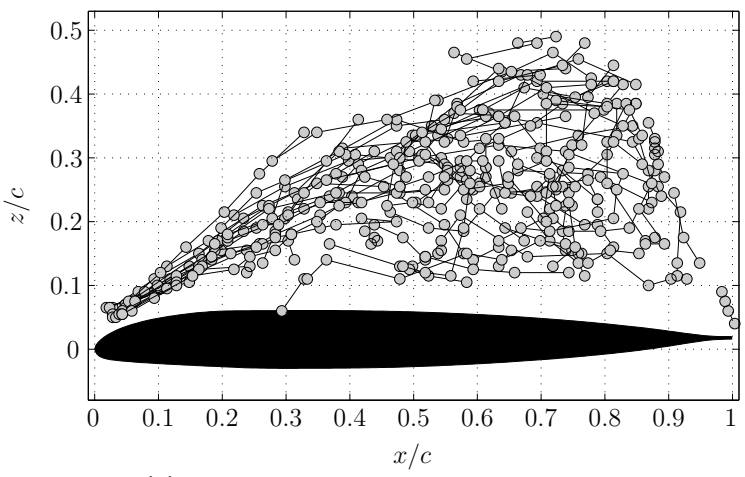

(e) $22^{\circ}>\alpha \geq 18^{\circ}$ on the downstroke

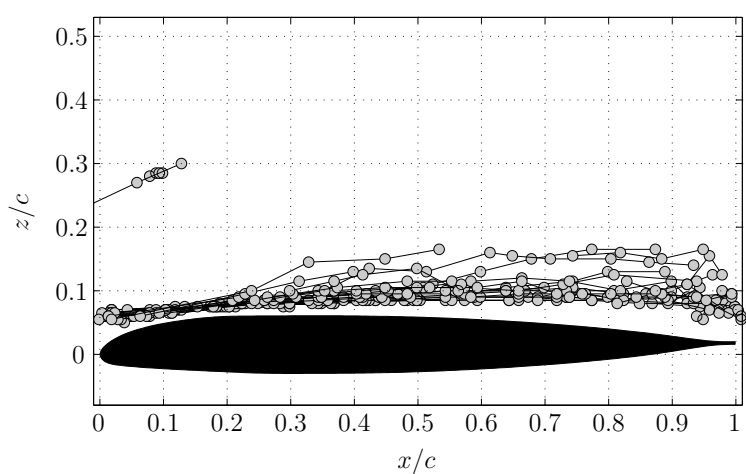

(b) $22^{\circ}<\alpha \leq 26^{\circ}$ on the upstroke

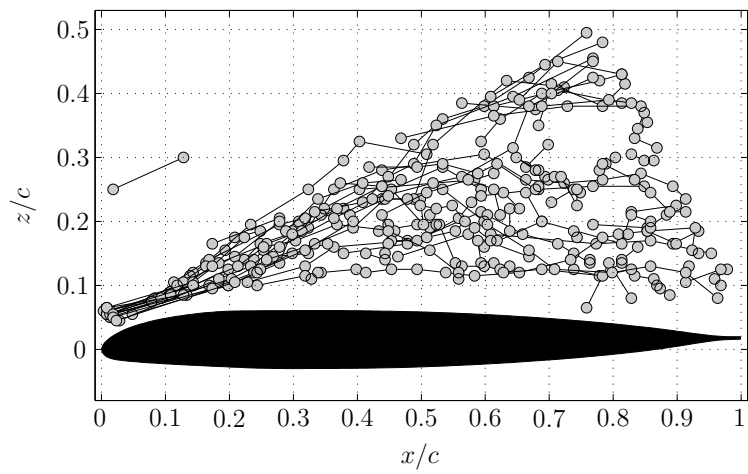

(d) $26^{\circ}>\alpha \geq 22^{\circ}$ on the downstroke

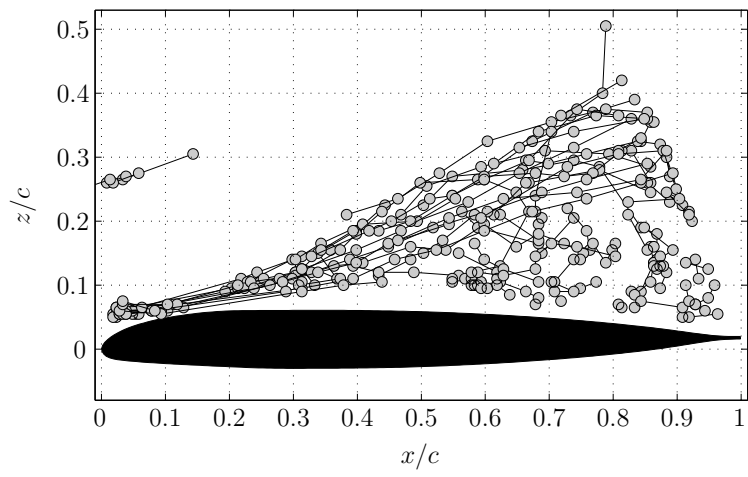

(f) $22^{\circ}>\alpha \geq 18^{\circ}$ on the downstroke

Figure 12. Detected cores and trajectories of vortices originating within different quarters of the arbitrary oscillation cycles A (left) and B (right) and $\alpha_{0}=18^{\circ}, \alpha_{1}=8^{\circ}, k=0.10$.

fluctuation of the aerodynamic coefficients is suppressed and the flow can reattach sooner.

\section{Conclusion and Future Perspectives}

The application of global time resolved imaging and an automated vortex identification algorithm yield insight into the prominent features of the dynamic stall process. Based on the location and evolution of the detected coherent structures, different stages were identified within the dynamic stall life cycle and the stall delay was assessed. The delay of dynamic stall was attributed to a finite formation time of the primary stall vortex and was observed to decrease with increasing unsteadiness. For the present data the motion induced speed of the leading edge at static stall angle appears to be the appropriate parameter to describe the unsteadiness with respect to stall delay. The induced leading edge stall vortex is assumed to be stronger for increasing nose speeds. The subsequent resulting stronger inviscid/viscous interactions cause the stall vortex to detache sooner from the surface. 


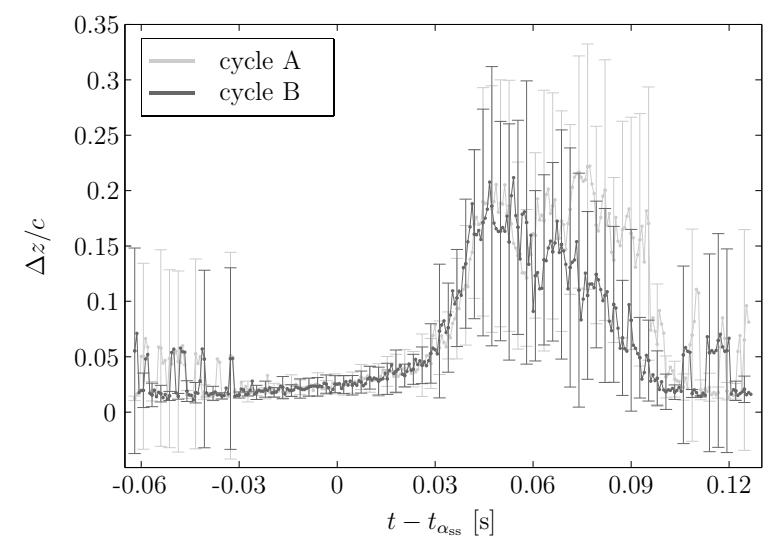

Figure 13. Average vertical displacement of the vortical structures versus time for two arbitrary oscillation cycles $\left(\alpha_{0}=18^{\circ}, \alpha_{1}=8^{\circ}, k=0.10\right)$.

A more systematic study, including an investigation of the Reynolds number effects would be necessary to sustain the above assumption and to understand the physical key mechanisms with regard to stall delay. Furthermore, higher spatial resolution measurements will yield more information about the strength and spatial scales of the coherent structures. Measurements of the three-dimensionality and spanwise extent of separation region are envisaged as well.

\section{Acknowledgements}

This work has been part of the DLR and ONERA joint project "Advanced Simulation and Control of Dynamic Stall" (SIMCOS). The dedicated support by J. Nuhn, T. Büte and J. Berhold from the DLR Institute of Aeroelasticity is gratefully acknowledged.

\section{References}

${ }^{1}$ Carr, L., McAlister, K., and McCroskey, W., "Analysis of the Development of Dynamic Stall Based on Oscillating Airfoil Experiments," Tech. rep., Ames Research center, NASA, 1977.

${ }^{2}$ McCroskey, W., "The Phenomenon of Dynamic Stall," Tech. rep., Ames Research Center, NASA, 1981.

${ }^{3}$ McCroskey, W., "Unsteady Airfoils," Ann. Rev. Fluid Mech., 1982.

${ }^{4}$ McCroskey, W. and Pucci, S., "Viscous-inviscid Interaction on Oscillating Airfoils in Subsonic Flow," AIAA Journal, 1982.

${ }^{5}$ Shih, C., Lourenco, L., Dommelen, L. V., and Krothapalli, A., "Unsteady Flow past an Airfoil Pitching at a Constant Rate," AIAA Journal, 1992.

${ }^{6}$ Shih, C., Lourenco, L., and Krothapalli, A., "Investigation of Flow at Leading and Trailing Edges of Pitching-up Airfoil," AIAA Journal, 1995.

${ }^{7}$ Doliganski, T., Smith, C., and Walker, J., "Vortex Interactions with Walls," Annu. Rev. Fluid Mech., 1994.

${ }^{8}$ Recent Developments in Theoretical and Experimental Fluid Dynamics, chap. The Dilemma of Defining a Vortex, Springer, 1979.

${ }^{9}$ Robinson, S., "Coherent Motions in the Turbulent Boundary Layer," Annual Review of Fluid Mechanics, 1991.

${ }^{10}$ Haller, G., "An Obective Defnition of a Vortex," Journal of Fluid Mechanics, 2005.

${ }^{11}$ Jeong, J. and Hussain, F., "On the Identification of a Vortex," Journal of Fluid Mechanics, 1995.

${ }^{12}$ Cucitore, R., Quadrio, M., and Baron, A., "On the Effectivenss and Limitations of Local Criteria for the Identification of a Vortex," European Journal of Mechanics - B/Fluids, 1999.

${ }^{13}$ Geissler, W., Dietz, G., Mai, H., Bosbach, J., and Richard, H., "Dynamic Stall and its Passive Control Investigationson the OA209 Airfoil Section," $31^{\text {st }}$ European Rotorcraft Forum.

${ }^{14}$ Mai, H., Dietz, G., Geiler, W., Richter, K., Bosbach, J., Richard, H., and de Groot, K., "Dynamic Stall Control by Leading Edge Vortex Generators," 62 $2^{\text {nd }}$ Annual Forum of the American Helicopter Society.

${ }^{15}$ Ewald, B., "Wind Tunnel Wall Correction," Tech. rep., AGARD, 1998.

${ }^{16}$ Hunt, J., Wray, A., and Moin, P., "Eddies, Stream, and Convergence Zones in Turbulent Flows," Tech. rep., Center for Turbulent Research, 1988.

${ }^{17}$ Chong, M., Perry, A., and Cantwell, B., "A General Classification of Three-dimensional Flow Fields," Physics of Fluids A, 1990.

${ }^{18}$ Michard, M., Graftieaux, L., Lollini, L., and Grosjean, N., "Identification of Vortical Structures by a non Local Criterion 
- Application to PIV Measurements and DNS-LES Results of Turbulent Rotating Flows," $11^{\text {th }}$ Conference on Turbulent Shear Flows.

${ }^{19}$ Jeon, D. and Gharib, M., "On the Relationship between the Vortex Formation Process and Cylinder Wake Vortex Patterns," J. Fluid Mech., 2004.

${ }^{20}$ Currier, J. and Fung, K., "Analysis of the Onset of Dynamic Stall," AIAA Journal, 1992.

${ }^{21}$ Visbal, M. and Shang, J., "Investigation of the Flow Structure around a Rapidly Pitching Airfoil," AIAA Journal, 1989. 